\title{
Uso de la cápsula endoscópica en niños con sangrado de tubo digestivo de origen desconocido
}

\author{
Dr. Rodrigo Hipólito-Cifuentes, ${ }^{*}$ Dr. Placido Espinoza-Rosas, ${ }^{* *}$ Dr. Pedro Salvador Jiménez-Urueta, ${ }^{* * *}$ \\ Dr. Luis Ariel Waller-González, ${ }^{* *}$ Dr. Ramón Alfredo Castañeda-Ortiz ${ }^{* *}$
}

\section{RESUMEN}

Intrucccion. La cápsula endoscópica (CE) es un dispositivo que permite examinar el intestino delgado. La cápsula es impulsada por la peristalsis a lo largo del tubo gastrointestinal y proporciona imágenes de video que son transmitidas por telerradiometría y captadas por una red de antenas adheridas al cuerpo. La cápsula puede detectar lesiones intestinales en pacientes con enfermedad de Crohn, con enfermedad celíaca; tumores malignos, alteraciones por el uso de antiinflamatorios no esteroides y síndromes que cursan con pólipos, malformaciones vasculares y hemangiomas. Presentamos la experiencia en nuestro hospital.

Material y método. En el Centro Médico Nacional 20 de Noviembre ISSSTE México de enero 2008 a marzo 2009 se realizó un estudio prospectivo no aleoatorizado, en pacientes con sangrado de tubo digestivo de origen desconocido cuyos estudios con panendoscopia y colonoscopia eran normales. Además de los parámetros habituales se registraron el diagnóstico inicial, el diagnóstico final, el tratamiento médico, el quirúrgico o ambos y la evolución.

Resultados. Fueron ocho pacientes entre nueve y 15 años de edad (media de 12). Seis masculinos y dos femeninos. Dos pacientes tenían hiperplasia linfoidea; un paciente tenía un hemangioma a $25 \mathrm{~cm}$ del Treitz. Dos pacientes, enfermedad de Crohn; un paciente pólipos en la porción media del íleon; un caso normal. En todos los casos excepto en el que el estudio fue normal, se resecaron las lesiones y se tomó una biopsia. La evolución fue satisfactoria en todos.

Discusión: La cápsula endoscópica es útil para detectar sangrado del intestino. Se usa cuando la colonoscopia y la panendoscopia son negativas y el paciente continúa sangrando; proporciona una imagen de certeza y permite evaluar la necesidad o no de un tratamiento quirúrgico.

Palabras clave: Sangrado de tubo digestivo, cápsula endoscópica, colonoscopia, enfermedad de Crohn, hiperplasia linfoidea.

\section{ABSTRACT}

Introductión. Capsule endoscopy (CE) is a wireless method for the examination of the intestine. The endoscopic capsule is propelled by peristalsis through the gastrointestinal tract. The video images it provides are transmitted by radiotelemetry to an aerial array attached to the body, which allows image capture. At present, it is considered the method of choice for the diagnosis of obscure gastrointestinal bleeding with 33 to $100 \%$ of diagnostic yield. CE can detect intestinal lesions in patients with Crohn's disease, caeliac disease, malignant tumors, anti-inflammatory drugs users, familial polyposis, hemangiomas, and vascular malformations. We present our experience with capsule endoscopy.

Material and method. In CMN 20 de Noviembre Hospital ISSSTE México we realize non randomized, prospective study, from January 2008 to March 2009 of patients with obscure gastrointestinal bleeding and a normal endoscopy. The study included primary diagnosis, end diagnosis, medical and/or surgical treatment, course.

Results. We studied 8 patients, between 9 and 15 years old, 6 males 2 females; 2 patients had lymphoid hyperplasia, 1 patient had a hemangioma at $25 \mathrm{~cm}$ distant from Trietz; 2 patients had Crohn's disease; 1 patient had polypos in the middle portion of the ileum, and in one case the study was normal.

Discussion. In patients with obscure gastrointestinal bleeding capsule endoscopy is important to identify the cause and location of the bleeding, it is special in patients in whom colonoscopy and endoscopy are normal. This method permits to decide whether surgical or medical treatment is indicated.

Key words: Bleeding of small bowel, capsule, endoscopy, colonoscopy, Crohn's disease, lymphoid hyperplasia.

* $\quad$ Residente de tercer grado de Cirugía Pediátrica

** Servicio de Endoscopia

*** Médico del Servicio de Cirugía Pediátrica

CMN 20 de Noviembre ISSSTE

Correspondencia: Dr. Pedro Salvador Jiménez-Urueta. CMN 20 de Noviembre ISSSTE. Félix Cuevas 540, Colonia del Valle. México 03100 DF.
Recibido: noviembre, 2009. Aceptado: enero, 2010.

Este artículo debe citarse como: Hipólito-Cifuentes R, EspinozaRosas P, Jiménez-Urueta PS y col. Uso de la cápsula endoscópica en niños con sangrado de tubo digestivo de origen desconocido. Acta Pediatr Mex 2010;31(2):55-59.

www.nietoeditores.com.mx 


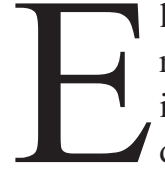
1 uso de la cápsula endoscópica (CE) es una modalidad de estudio que ha generado gran impacto en los últimos años ${ }^{1-3,11}$ en el abordaje diagnóstico de pacientes con hemorragia digestiva. Desde su aprobación por la FDA en el año 2001, se han realizado más de 250,000 estudios en todo el mundo y es probable que en el futuro próximo, su uso para el tratamiento de las enfermedades digestivas sea más difundido. La ventaja principal de este método sobre los demás radica en que permite visualizar la totalidad del intestino delgado, cualidad de la que carecen los métodos endoscópicos que se utilizan en el estudio de la hemorragia gastrointestinal ${ }^{4-6}$. La cápsula endoscópica, puede visualizar hasta $120 \mathrm{~cm}$ distales al ángulo de Treitz ${ }^{7}$ en tanto que la colonoscopia sólo hasta $60 \mathrm{~cm}$ de íleon. La CE permite realizar un estudio mínimamente invasivo, inocuo, que no requiere sedación.

\section{Breve reseña histórica}

Los primeros ensayos de fabricación de la cápsula endoscópica (CE) comenzaron a principios de la década de los años noventa del siglo XX. Dos grupos independientes trabajaron en sus primeros diseños. Gabriel Iddan, ingeniero mecánico israelí, durante sus años sabáticos se dedicó a idear sistemas de visualización con microcámaras para uso militar. Paul Swain, gastroenterólogo inglés, realizaba por entonces actividades similares con la idea de visualizar el intestino delgado. En 1997, los dos grupos se unieron para trabajar conjuntamente. En 1999, se realizó el primer estudio en humanos. Paul Swain ingirió dos CE en dos días sucesivos y así se obtuvieron las primeras imágenes de intestino delgado en humanos. En el año 2000, la revista Nature publicó las primeras experiencias en diez voluntarios lo que generó gran expectativa. A principios del año 2001 la revista New England Journal of Medicine, publicó cuatro casos de sangrado de origen oscuro; su localización se logró con la cápsula endoscópica. En agosto de 2001, la FDA aprobó el uso de la CE para el estudio del sangrado de origen desconocido. En el año 2005, la FDA aprobó un nuevo modelo de CE para el estudio del tubo digestivo.

\section{Características generales}

La CE mide $26 \mathrm{~mm}$ de largo y $11 \mathrm{~mm}$ de ancho, muy similar al tamaño del extremo distal de un endoscopio convencional. El ángulo de visualización es de 140 grados y el poder de resolución es x8 (Figura 1). Registra

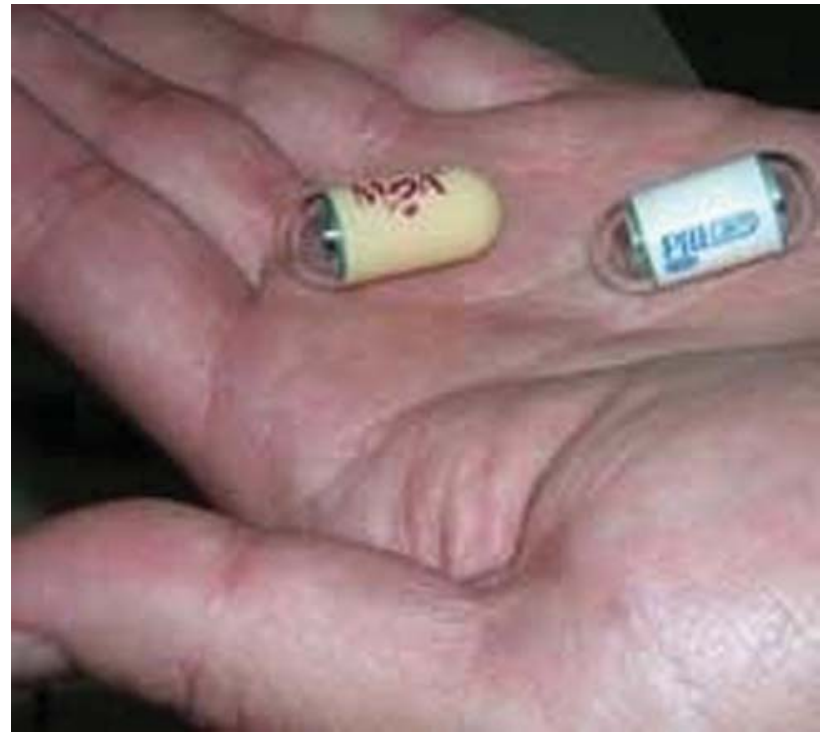

Figura 1.

dos imágenes por segundo durante ocho horas lo que da aproximadamente 57,000 imágenes. La cápsula endoscópica transmite las imágenes a través de un sistema de tele radiofrecuencia que son captadas por electrodos adheridos al abdomen del paciente. Estos electrodos, por un sistema de cables, transmiten las imágenes a un disco rígido portátil de 5 gigabytes que almacena las imágenes del estudio. La CE es ingerida por el paciente con un vaso de agua. El paciente debe estar en ayuno doce horas. Se sugiere la preparación del intestino con polietilenglicol (2 a 4 litros) o con fosfato sódico. El desplazamiento de la cápsula por el tubo digestivo se debe a los movimientos peristálticos gastrointestinales fisiológicos y no se requiere insuflación de aire. El uso de pro-cinéticos (metoclopramida, eritromicina, cisaprida) permite una mejor visualización del ciego. Una vez ingerida la cápsula, el paciente puede realizar sus actividades diarias habituales durante las ocho horas que dura el estudio. Puede ingerir una comida líquida dos horas después de iniciado el estudio y una comida semisólida luego de cuatro horas de la ingestión de la cápsula. Al final del recorrido la cápsula es eliminada por las heces; no es reutilizable. Tras la conclusión de la prueba, las imágenes obtenidas, se procesan con un ordenador, que permite seleccionar las más representativas y estudiarlas con detalle. En la actualidad existe un software para localizar el sangrado, capaz de identificar áreas con mayor número de pixeles de color rojo. Este software agiliza el estudio. 
Importa señalar que 28 a $70 \%$ de las causas de sangrado de origen obscuro obedecen a lesiones visibles por endoscopia convencional.

Por lo tanto, conviene repetir el procedimiento endoscópico antes de indicar el estudio del intestino delgado con la CE. Los estudios radiológicos con bario no logran identificar lesiones planas, ni mínimamente elevadas como las angiodisplasias, por lo que tienen poca indicación. La arteriografía mesentérica y la gammagrafía con hematíes marcados también se han utilizados para identificar el sitio sangrante, pero sólo son útiles en el momento del sangrado activo. Por lo tanto, la tasa de diagnósticos de estas técnicas se estimen en 34\% para la arteriografía y en $51 \%$ para la gammagrafía. Las cápsulas endoscópicas permiten visualizar gran parte del intestino delgado y alcanzan el íleon en el 100\% de los casos ${ }^{8-10 .}$

\section{MATERIAL Y MÉTODO}

Se estudiaron ocho pacientes (seis del sexo masculino y dos del femenino) de nueve a 15 años de edad con sangrado de tubo digestivo de origen desconocido. Todos tenían biometría hemática, química sanguínea, examen general de orina y serología para enfermedad celíaca (anticuerpos antigliadina, antiendomisio y antitransglutamina), que fueron negativos. Se descartó en todos infección por Helicobacter pylori mediante la prueba de aliento. En todos se había realizado al menos la endoscopia peroral, la colonoscopia y el ultrasonido abdominal que fueron normales. El estudio de tránsito gastrointestinal también fue normal; no había estenosis intestinales que contraindicaran el uso de la CE. Todos los pacientes recibieron la cápsula endoscópica por la boca. El consentimiento informado siempre fue firmado por uno de los padres. Los pacientes fueron cuidadosamente monitorizados para detectar efectos adversos. Se administró la cápsula endoscópica (M2A o Pillcam de Given Imaging) (Figura 1) y se registró la grabación a través de electrodos externos colocados en la pared abdominal y a través de su sistema Holter. Ningún paciente tuvo problemas para deglutir la cápsula. La CE fue excretada sin problemas; ningún paciente sufrió dolor abdominal durante el estudio.

\section{RESULTADOS}

En siete de los ocho pacientes se obtuvo un diagnóstico definitivo. Un paciente tenía hiperplasia linfoidea con hemangiomas a $25 \mathrm{~cm}$ del ángulo de Treitz (Figura 2). Otro paciente con hiperplasia linfoidea tenía enfermedad de Crohn (Figura 5). Dos pacientes tenían hiperplasia linfoidea e inflamación inespecífica causante del sangrado del tubo digestivo. En un paciente se hallaron pólipos en la porción media del íleon (Figura 3). En un paciente el estudio fue normal. Un paciente tenía una malformación arteriovenosa en la porción media del íleon (Figura 4). En un paciente se hallaron erosiones; las biopsias correspondientes revelaron la enfermedad de Crohn. Los siete pacientes con estudio anormal fueron operados para resección de las lesiones, para toma de biopsia o ambos procedimientos. La panendoscopia y colonoscopia fueron negativas.

En dos de nuestros pacientes se intento mediante laparoscopia corroborar el diagnóstico, pero no se observó lesión alguna en la superficie intestinal; por lo tanto consideramos de suma utilidad la utilización de la CE.

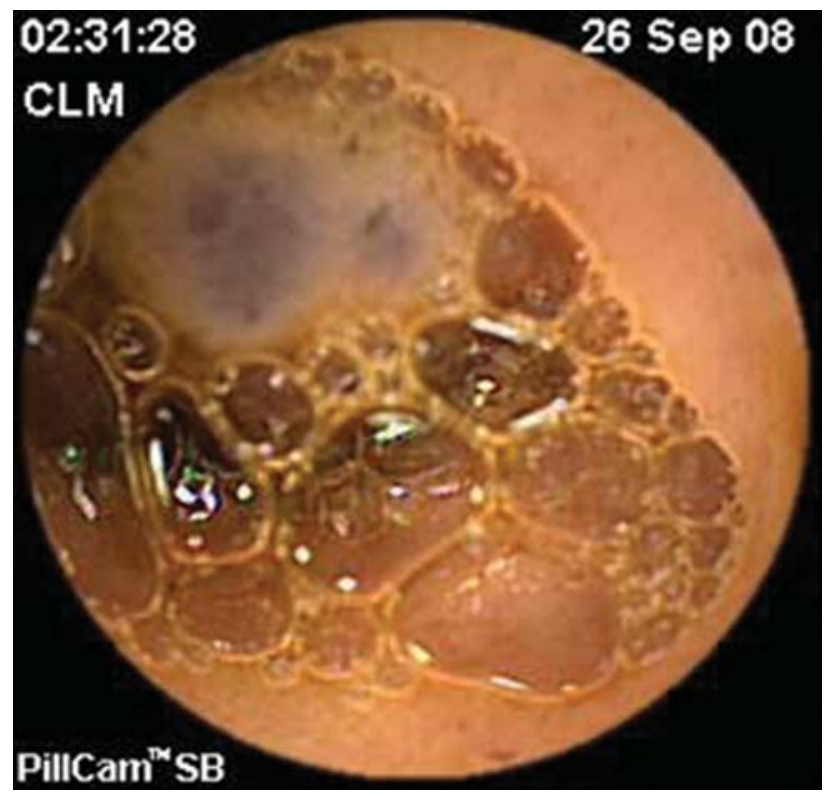

Figura 2. Se observa el hemangioma.

\section{DISCUSIÓN}

En niños con sangrado de tubo digestivo de origen desconocido es fundamental identificar el sitio del problema, para orientar adecuadamente el tratamiento.

En pacientes con persistencia de hemorragia gastrointestinal se justifica usar la cápsula endoscópica. Se ha utilizado la CE a nivel mundial incluso en niños de 10 


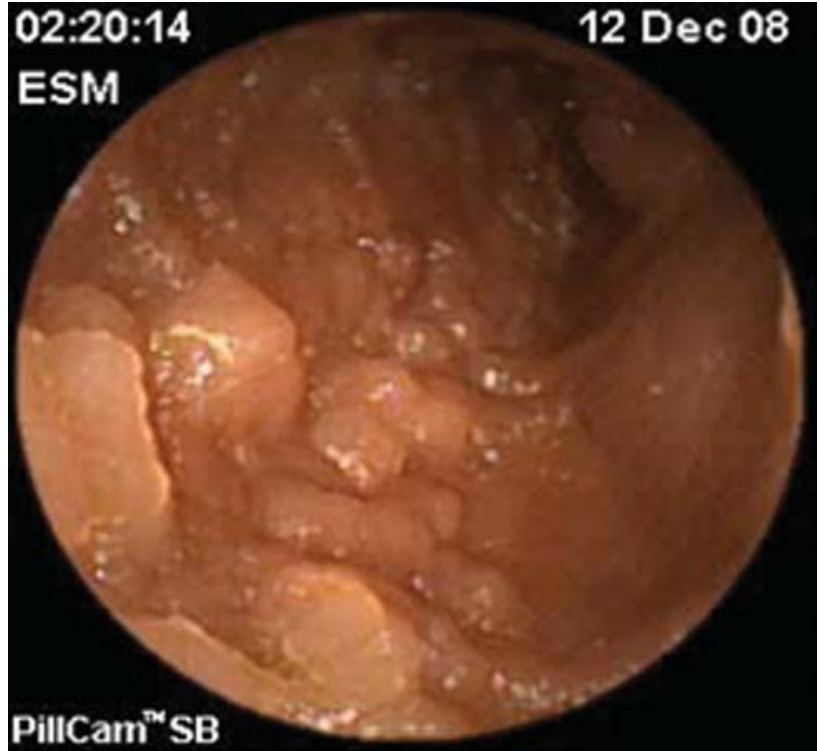

Figura 3. Pólipos en la mucosa.

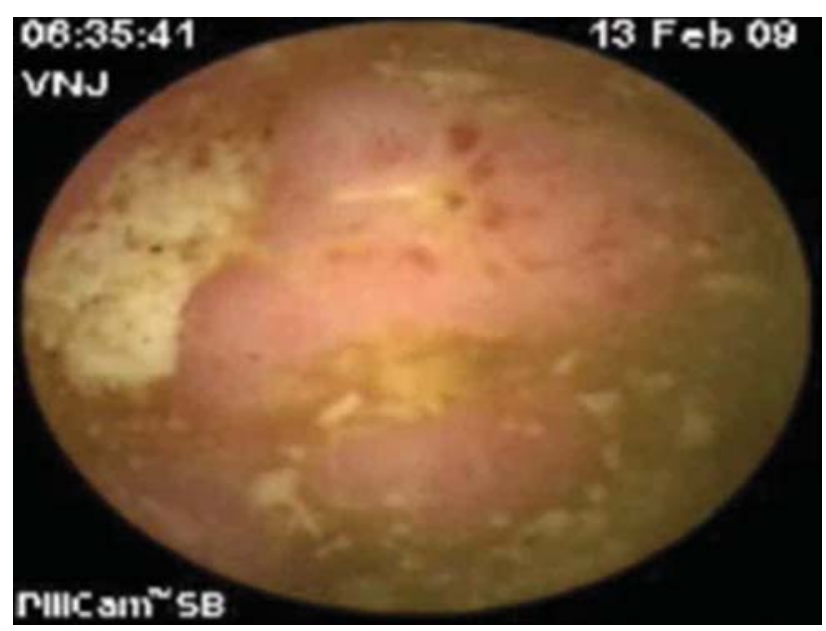

Figura 4. Imágenes de angiodisplasia intestinal.

$\mathrm{kg}$ de peso ${ }^{13-16}$. En la literatura se menciona el uso de sedantes para la realización del estudio ${ }^{15,16}$; sin embargo, su empleo tiene complicaciones, una de las cuales es la retención de la cápsula, lo que requiere una intervención quirúrgica, si después de diez días no se ha evacuado. En nuestros pacientes no hubo complicaciones; no recibieron sedantes. Se les dio seguimiento con estudio fluoroscópico en las siguientes $48 \mathrm{~h}$. La CE es muy útil en pacientes en quienes se desconoce el sitio del sangrado intestinal y tiene mayor utilidad cuando se sospecha que el problema puede estar en el intestino medio. Las imágenes que se obtienen

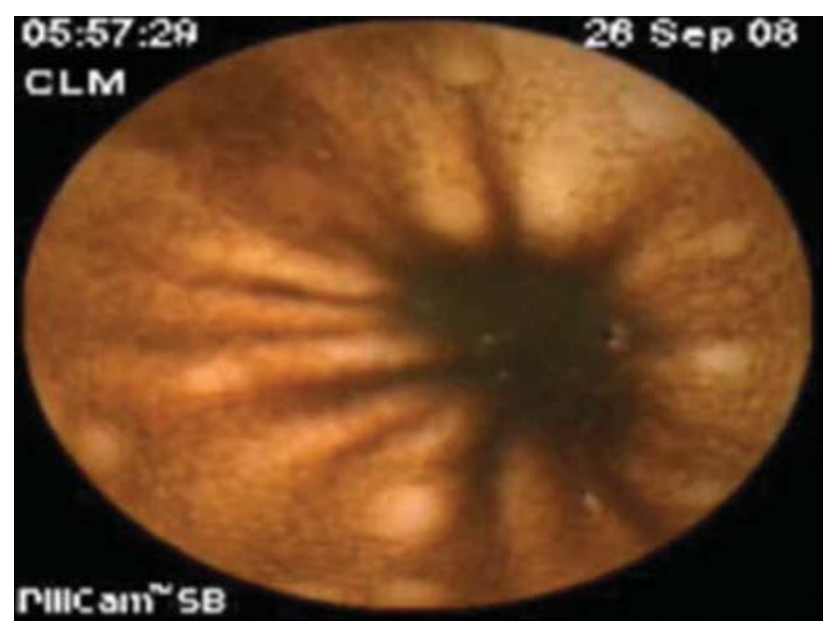

Figura 5. Imágenes de hiperplasia linfoidea con lo que se demostró enfermedad de Crohn.

son nítidas y permiten hacer un diagnóstico de presunción muy cercano al que da el estudio patológico.

Por ser lesiones difusas en nuestros casos, se requirió tomar biopsias intestinales, las cuales corroboraron el diagnóstico.

\section{CONCLUSIÓN}

En la literatura nacional e internacional no encontramos artículos que mencionen casos como los nuestros, cuyos diagnósticos se lograron con el uso de la CE. La utilidad presente y futura que tiene el estudio es innegable. Se debe seleccionar con cuidado los pacientes y hacer un uso racional del recurso, sobre todo cuando los métodos tradicionales no logran el diagnóstico y persisten el dolor, el sangrado o ambos.

\section{BIBLIOGRAFÍA}

1. Croffie JM, Fitzgerald JF, Chong SK. Recurrent abdominal pain in children - A retrospective study of outcome in a group referred to a pediatric gastroenterology practice. Clin Pediatr (Phila). 2000;39:267-71.

2. Alfven G. One hundred cases of recurrent abdominal pain in children: diagnostic procedures and criteria for a psychosomatic diagnosis. Acta Pediatric. 2003;92:43-9.

3. Nadler M, Bardan E. Capsule endoscopy in Crohn's disease. Med Assoc J. 2005;7:262-3.

4. Fireman Z, Mahajna E, Broide E, Shapiro M, Fich L, Sternberg A, et al. Diagnosing small bowel Crohn's disease with wireless capsule endoscopy. Gut. 2003;52:390-2.

5. Herrerías JM, Cañedo A, Rodríguez-Téllez M, Pellicer F, Herrerías JM Jr. Capsule endoscopy in patients with suspec- 
ted Crohn's disease and negative endoscopy. Endoscopy. 2003;35:564-8.

6. Melmed GY, Lo SK. Capsule endoscopy: practical applications. Clin Gastroenterol Hepatol. 2005;3:411-22.

7. Delvaux M, Fassler I, Gay G. Clinical usefulness of the endoscopic video capsule as the initial intestinal investigation in patients with obscure digestive bleeding: validation of a diagnostic strategy based on the patient outcome after 12 months. Endoscopy. 2004;36:1067-73.

8. Eliakim R, Fischer D, Suissa A, Yassin K, Katz D, Guttman $\mathrm{N}$, et al. Wireless capsule video endoscopy is a superior diagnostic tool in comparison to barium follow-through and computerized tomography in patients with suspected Crohn's disease. Eur J Gastroenterol Hepatol. 2003;15:3637.

9. Caunedo Álvarez A, Herrerías Gutiérrez JM. Role of endoscopic capsule in the diagnosis of digestive diseases. Med Clin (Barc). 2005;124:427-33.
10. Argüelles-Arias F, Caunedo A, Romero J, Sánchez A, Rodríguez-Téllez M, Pellicer FJ, et al. The value of capsule endoscopy in pediatric patients with a suspicion of Crohn's disease. Endoscopy. 2004;36:869-73.

11. Lewis B, Goldfarb N. Review article: the advent of capsule endoscopy-a not-so-futuristic approach to obscure gastrointestinal bleeding. Aliment Pharmacol Ther. 2003;17:1085-96.

12. Iddan G, Meron G, Glukhovsky A, Swin P. Wireless capsule endoscopy. Nature. 2000;405:417-23.

13. Aabakken L, Scholz T, Ostensen A, Emblem R, Jermstad T. Capsule endoscopy is feasible in small children. Endoscopy. 2003;35:798-806.

14. Sultan S, Dobozi B, Palmer P, Treem W, Agrawal N. Assisted wireless capsule endoscopy in a six-year-old with abdominal pain and obscure Gl bleeding. Gastrointest Endosc. 2003;57:AB127-134.

15. Mallet E, Cron J, Stoller J. Wireless-capsule video-endoscopy: preliminary results in children. Arch Pediatr. 2003;10:244-5. 